\title{
A Novel Accurate Localization Method of Vehicle-logo
}

\author{
Jin Liu ${ }^{1,2, a}$, De Cai ${ }^{1,2, b}$, Zhonghan Shi ${ }^{1,2, c}$ and Jiahao Deng*1, 2, d \\ ${ }^{1}$ Key Laboratory of Electromechanical Dynamic Control, Beijing, China, 100081 \\ ${ }^{2}$ School of Mechatronical Engineering, Beijing Institute of Technology, Beijing, China, 100081

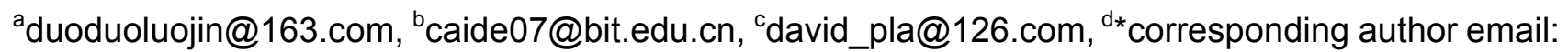 \\ bitdjh@bit.edu.cn
}

Keywords: Vehicle-logo, Accurate localization, Gradient angle histogram analysis, Local self-adaptive threshold.

\begin{abstract}
Aiming at the problem lied in existed methods which cannot suppress grille background completely, a novel method of vehicle-logo accurate localization was proposed. Firstly color invariants and prior information are used for fast localizing license plate in RGB color space directly; and then process fine localization of the vehicle-logo based on coarsely localized results. Background noises are secondarily suppressed based on gradient angle histogram analysis and local self-adaptive threshold treatment. Numerous experiments show that this method possesses not only high accuracy but also high robustness in localization, as compared to classic template matching. Besides, the proposed method satisfies the requirement of real-time performance.
\end{abstract}

\section{Introduction}

Vehicle recognition has long been one of the hot research topics in the field of intelligent transport system (ITS). A vehicle-logo (VL) usually contains important information on vehicle model and manufacturers, and is not easy to be counterfeited or misappropriated. Thus in recent years, using VL to assist improving recognition rate of vehicle recognition system has become a new research direction [1].

Accuracy of VL localization can directly affect the recognition result, therefore a novel method to accurately locate VL is proposed in this paper. In this method, the first step is coarse localization of VL by extracting color invariants and using prior knowledge on the relative position of the logo to the license plate (LP). Background grille suppression, projective histogram of edge gradients and mathematical morphology method are then executed to gradually realize fine localization of VL. Further examination and correction of fine localization result are carried out with analysis of edge gradient angle histogram and treatment of local self-adaptive threshold value. Localization of VL with high accuracy and robustness are generated as a result.

\section{Coarse Localization of VL}

Most of vehicle license plates in China are blue in color, which usually appears distinct from the background. Consequently, to locate the VL in a colored image, a very effective way is recognizing the LP by color, followed by coarse localization utilizing prior knowledge of relative positions. Most researchers use Hue $(\mathrm{H})$ and Saturation $(\mathrm{S})$ for color recognition through color space transformation, as these two parameters are more stable with regard to illumination conditions [2, 3]; but the transformation process affects real-time quality of this method. In this paper, two color invariants are extracted directly from the RGB space according to eq. 1, and the blue LP is examined with threshold values defined in eq. 2; then the prior knowledge that a VL (usually symmetric) locates directly above the medial axis of a LP is adopted to identify the rough position of the VL. As such the coarse localization algorithm is simplified and runs faster [4]. The coarse localization process of VL is shown in Fig.1. 


$$
\begin{aligned}
& \left\{\begin{array}{l}
\text { Ratio }_{1}=\frac{\omega_{B}-\omega_{R}}{\omega_{B}}=1-\frac{k_{R}}{k_{B}} \\
\text { Ratio }_{2}=\frac{\omega_{B}-\omega_{G}}{\omega_{B}}=1-\frac{k_{G}}{k_{B}}
\end{array}\right. \\
& \Phi\left(\text { Ratio }_{1}, \text { Ratio }_{2}\right)=\left\{\left(x_{1}, x_{2}\right) \mid x_{1} \& x_{2} \in(0.25,+\infty)\right\}
\end{aligned}
$$

Where $x_{1}$ and $x_{2}$ denote the variable range of Ratio ${ }_{1}$ and Ratio ${ }_{2}$ respectively.

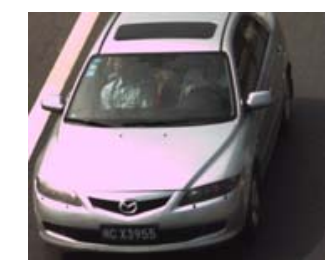

Unprocessed Original Colored Image

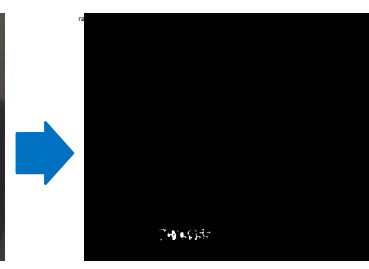

Color Extraction

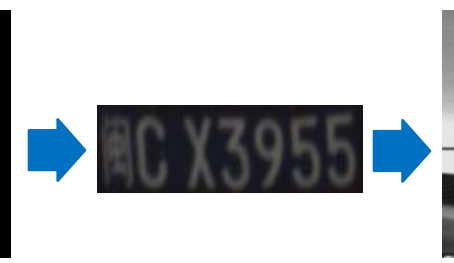

Location of License Plate

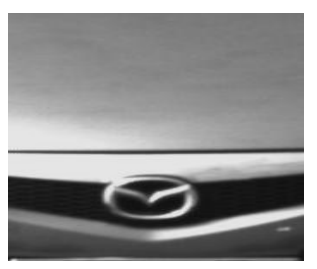

Coarse Localiaztion Region

Fig. 1 Coarse Localization Process of Vehicle-logo

\section{Fine Localization of VL}

Background Texture Suppression. Intake grilles on vehicle can impact fine localization and recognition of VL. Thus in this paper, a VL texture measurement is defined to determine appropriate edge detection methods for effectively suppressing background noises.

The proposed method detects horizontal and vertical edges in a measurement window with parameters $[1 / 3 \mathrm{~m}, 2 / 3 \mathrm{~m}]$ and $[1 / 6 \mathrm{n}, 1 / 3 \mathrm{n}](\mathrm{m}, \mathrm{n}$ are the height and width of the coarse localization region), a constant threshold value of 5 is chosen to binarize the resulted image. After then compute the sum of pixels' values $\mathrm{X}$ and $\mathrm{Y}$ for horizontal and vertical edge detection images respectively. As shown in Table 1, if texture measurement $\mathrm{X}>\mathrm{Y}$, the background texture is identified as horizontal, otherwise as vertical. If $\mathrm{X} \approx \mathrm{Y}$, the background texture is identified as mesh-like. Horizontal and vertical grilles are the focus in this paper as they are most commonly seen.

\begin{tabular}{|c|c|c|c|c|c|c|c|}
\hline \multirow{2}{*}{$\begin{array}{c}\text { Coarse Localization } \\
\text { Region } \\
\end{array}$} & \multicolumn{3}{|c|}{ Measurement Results } & \multirow{2}{*}{$\begin{array}{c}\text { Coarse Localization } \\
\text { Region }\end{array}$} & \multicolumn{3}{|c|}{ Measurement Results } \\
\hline & $\mathrm{X}$ & $\mathrm{Y}$ & Texture & & $\mathrm{X}$ & $\mathrm{Y}$ & Texture \\
\hline & 206 & 0 & Horizontal & & 201 & 354 & Vertical \\
\hline & 49 & 0 & Horizontal & 늘 & 0 & 136 & Vertical \\
\hline
\end{tabular}

Table 1 Background Texture Measurement

According to detection result of background texture, conduct edge detection using complementary single Sobel operator can suppress influences of background textures. Then fill gaps and reconnect disconnected parts with morphology transformation to obtain the precise VL region. The suppression process is shown in Fig. 2. 


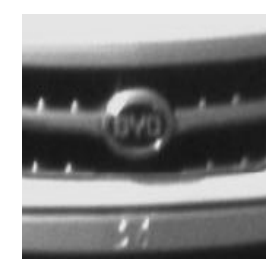

Region of Coarse Localization

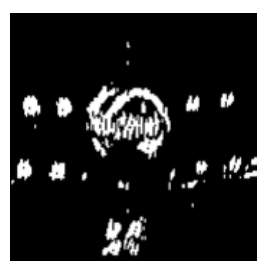

Edge Detection Using Vertical Sobel Operator

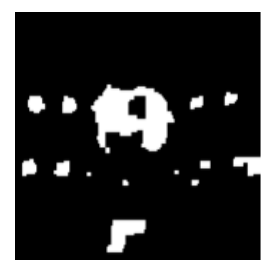

After Closed Operation

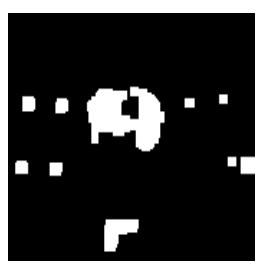

After Open Operation

Fig. 2 The Process of Background Texture Suppression

Fine Localization Based on Projective Histogram. For fine localizing of VL, horizontal projective histogram is used to determine upper and lower boundaries of the logo, while its left and right boundaries are determined with vertical projective histogram. Fig. 3 below displays projective histograms of the resulted image after background texture suppression.

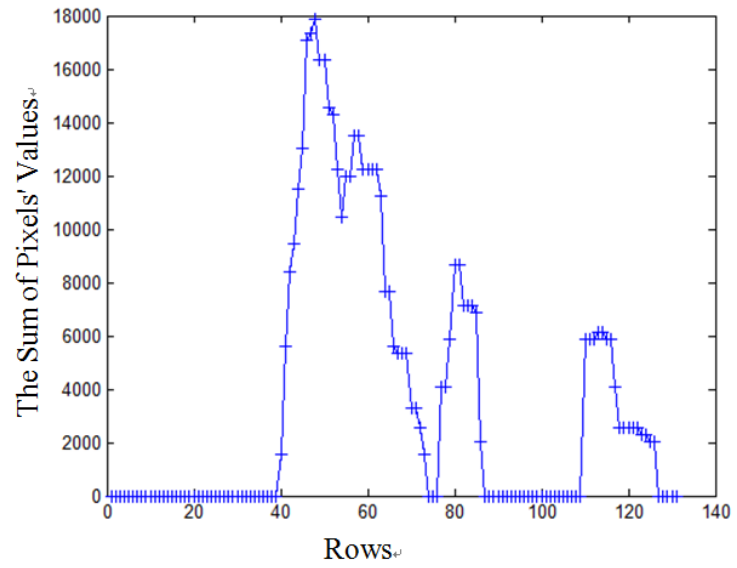

a) Horizontal Projective Histogram

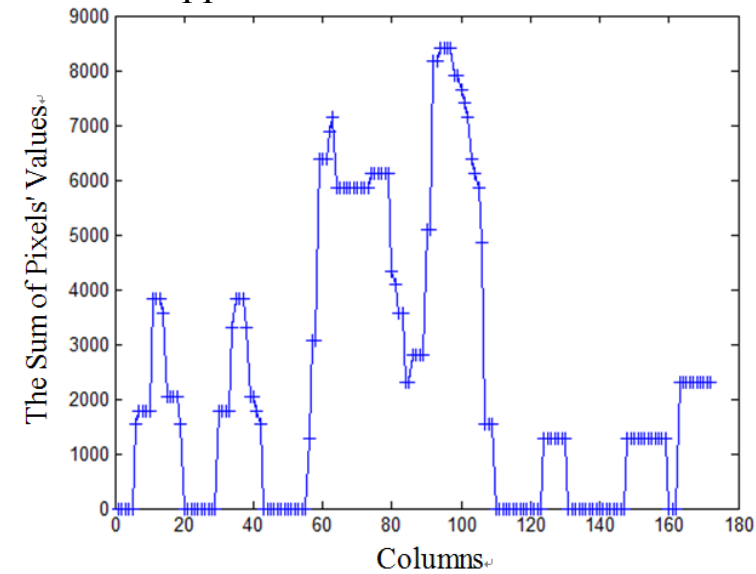

b) Vertical Projective Histogram

Fig. 3 Projective Histograms

\section{Further Examination and Correction of Fine localization result}

Under most conditions, the obtained logo region after coarse to fine processing still contains some parts of the grille background, which will affect recognition rates in subsequent procedures. Therefore, this paper proposes a further suppression measure, which uses gradient angle histogram analysis and local self-adaptive threshold value treatment to check the validity of fine localization region row-by-row (or column-by-column), and correct where appropriate.

Fig. 4 displays the examination and correction processes. As VL is symmetric, gather edge gradient angle histogram statistics (Fig. 4a) inwardly along four distinct directions (up, down, left, right) row-by-row (or column-by-column). Take horizontal grilles as an example, to avoid angles at edges, edge gradient angle statistics within the ranges $\left[-60^{\circ},-10^{\circ}\right]$ and $\left[10^{\circ}, 60^{\circ}\right]$ are mainly concerned. If statistics in the two ranges are both positive and do not differentiate significantly from each other, the currently examined rows/columns are identified as having a good left-right/up-down symmetry, and are thus reserved as they contain parts of the VL. Otherwise if any of the two conditions is not met, the examined rows/columns are regarded as asymmetric in left-right/up-down directions, and are thus eliminated since they are non-VL regions. When the VL region is treated as described above (see Fig. 4b), consider that in the image, real factors like surrounding illumination and shooting angles can cause brightness nonuniformity on the VL, and treat the grayscale image of current regions with self-adaptive mean filter (of window size $15 \times 15$ ). This guarantees a complete extraction of VL features. The last step is to bidirectionally project the processed binary image (Fig. 4c) for obtaining the further-optimized VL boundaries (Fig. 4d). 


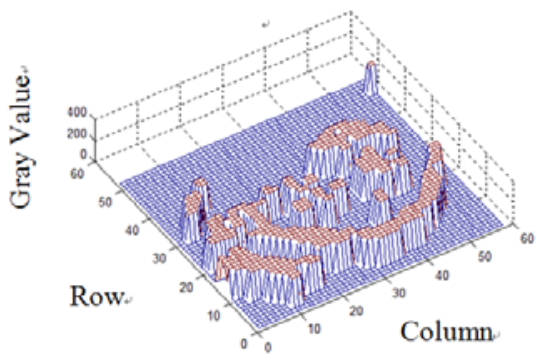

a. Original Fine Localization Region

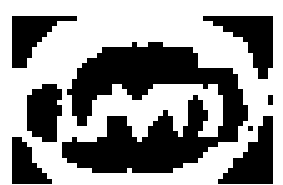

c. After Local Self-adaptive Threshold Filtering

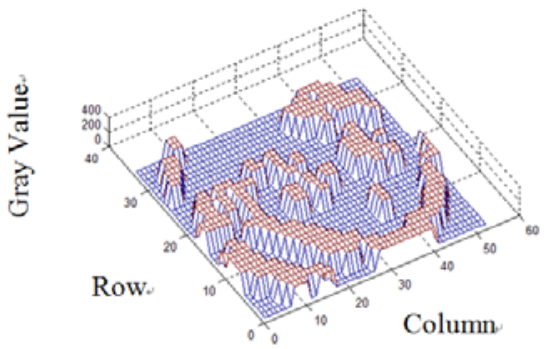

b. After the Treatment of Gradient Angle Histogram

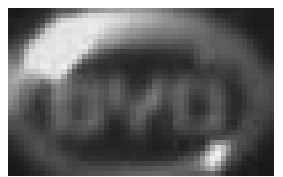

d. The Resulted Image

Fig. 4 Procedures to Examine and Correct Fine Localization Result

\section{Results and Analysis}

In this paper, 67 logo images of 5 vehicle brands (Volkswagen, BYD, Mazda, Nissan and Hyundai) are used in experiments. All images are actual shootings from a road traffic monitoring system. Original vehicle images are around $1000 \times 1000$ pixels in size. The VL database includes conditions of different weather conditions, various illumination directions, diverse shooting directions and dirty VLs. The rich variety of samples helps examine the robustness of the algorithm, which is executed on the MATLAB R2012b platform (Windows 7 Ultimate, 64-bit), with hardware conditions: IntelI CoreI i5-3210M CPU @ 2.50GHz, RAM 4.00GB.

Quantitative analysis of experimental results employs locating accuracy and average locating time as the two main parameters, which are conventional in VL localization researches [5]. Table 2 and Fig. 5 compare accuracies and time taken of the proposed method to those of the classical template matching (TM) method.

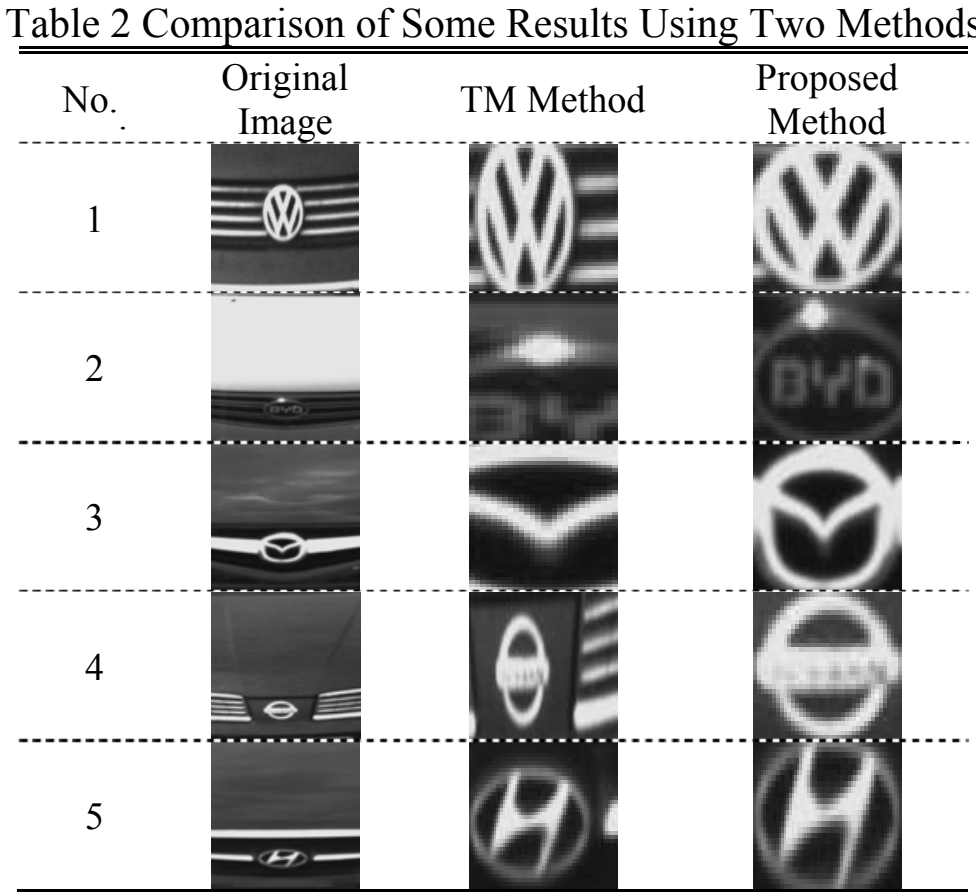

In this experiment, the proposed method generated a 100\% locating accuracy, which implies all VLs are extracted completely and accurately. However for the TM method, 28 results (which is 
occupied $41.79 \%$ ) had significant locating errors, and 7 results $(10.45 \%$ of the total) were incompletely located. Moreover, TM method assumes intake grilles in the background have little or no changes in the horizontal direction, while the proposed method does not require such assumption. Therefore the later method has stronger robustness.

To compare the average locating time of the two methods, both methods were executed 12 times respectively to treat the coarse localization region of the same image:

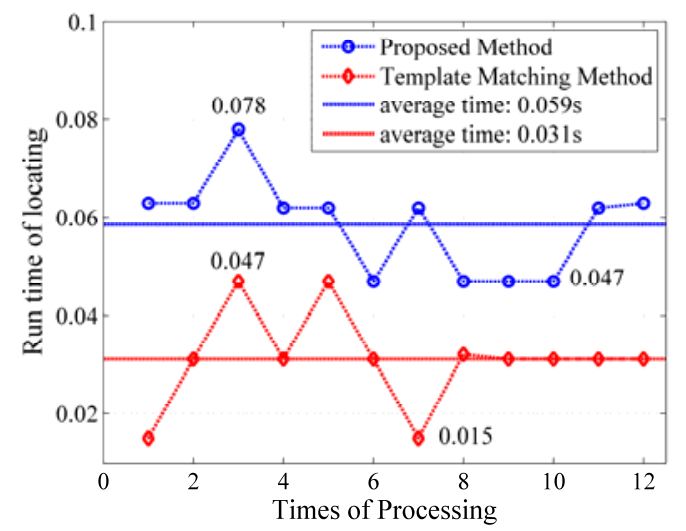

Fig. 5 Comparison of the Average Locating Time of the Two Methods

From above results, the average locating time of TM method is around $0.04 \mathrm{~s}$, and the locating time of proposed method varies from $0.05 \mathrm{~s}$ to $0.08 \mathrm{~s}$, this still satisfies real-time requirement.

\section{Summary}

This paper introduces a highly accurate and robust VL localization method. In this method, two color invariants of VL's background color are extracted under the RGB model, thus to achieve fast and highly robust coarse localization of VL. After fine localization of VL, the edge gradient angle histogram analysis, followed by the treatment of local adaptive threshold, is suggested to validate and correct localization result for further increasing the locating accuracy. Experimental results indicate that the proposed method has high locating accuracies, and produces localization results with effectively complete information of VLs; results are also strongly robust against changing in VL sizes, as well as dimming and non-uniform illuminations. Moreover, despite the longer time taken for localization as compared to the classical TM method, the proposed method still satisfies real-time performance requirement.

Since yet there exists no standard database for VLs, and the self-built VL database used in this paper is currently small in size, it must be expanded in the future in order to improve the universality of the proposed localization algorithm.

\section{References}

[1] Yang W, Yang G, Zheng X, et al. An improved vehicle-logo localization algorithm based on texture analysis[C]// Computer Science and Information Processing (CSIP), 2012 International Conference on IEEE, 2012:648-651.

[2] Shi X, Zhao W, Shen Y. Automatic license plate recognition system based on color image processing[J]. Lecture Notes in Computer Science, 2005, 3483: 1159-1168.

[3] Lee E R, Kim P K, Kim H J. Automatic recognition of a car license plate using color image processing. Proc. IEEE Int. Conf. Image Process.[C]. 1994, 2.

[4] De C, Zhonghan S, Jin L, et al. Robust and fast license plate detection based on the fusion of color and edge feature. International Conference on Optoelectronic Technology and Application 2014 [C]. Beijing China, May 2014, oral presentation, in press. 
[5] Qiongyun W, Zhifang L, Fei X. Real-time Vehicle-logo Localization and Recognition Based on Symmetry [J]. Journal of System Simulation, 2009, 21 (4) : 1095-1100. (in Chinese) 